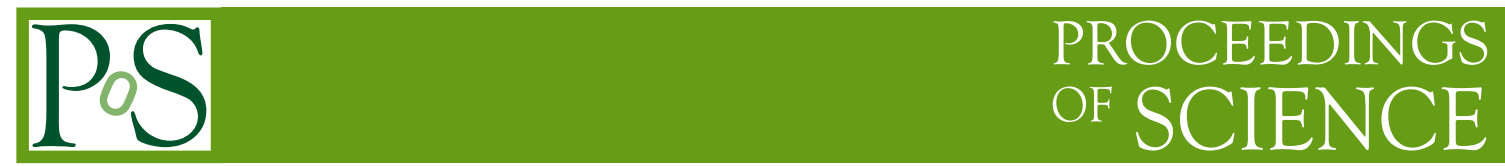

\title{
Review of indirect charged Higgs searches at B factories
}

\author{
Maria Różańska* \\ H. Niewodniczański Institute of Nuclear Physics, Polish Academy of Science \\ E-mail: maria.rozanska@ifj.edu.pl
}

The latest measurements of $B$ physics observables, sensitive to charged Higgs contributions, are reviewed. The results are based on large data samples collected with the Belle and BaBar detectors at the $\Upsilon(4 S)$ resonance in the $e^{+} e^{-}$asymmetric colliders, KEKB and PEP-II. Some implications for constraining the charged Higgs sector are discussed within the context of the Type-II two Higgs doublet model.

Third International Workshop on Prospects for Charged Higgs Discovery at Colliders - CHARGED2010, September 27-30, 2010

Uppsala Sweden

\footnotetext{
* Speaker.
} 


\section{Introduction}

Charged Higgs bosons $\left(H^{ \pm}\right)$are predicted in many well-motivated extensions of the standard model (SM). The expected couplings to the standard model particles are large enough to enable observations of a $H^{ \pm}$both directly at the LHC and indirectly in flavor observables.

The simplest class of the models with the extended Higgs sector are two-Higgs-doublet models (2HDM). Among them the Type-II 2HDM is of special importance, as it has the same couplings of the $H^{ \pm}$to fermions as the minimal supersymmetric extension of the SM (MSSM) [1]. In the Type-II 2HDM the two Higgs doublets couple separately to the down-type quarks and the up-type quarks. The coupling of $H^{ \pm}$to a fermion pair $f_{u} f_{d}$ is proportional to $m_{f} \tan \beta$, where $m_{f}$ denotes a mass of a down-type quark or a charged lepton, and $\tan \beta \equiv v_{2} / v_{1}$ is the ratio of the vacuum expectation values of the two Higgs fields.

$B$-meson decays offer an interesting laboratory for indirect searches of $H^{ \pm}$. The relatively large $b$-quark mass both enhances the $H^{ \pm}$couplings and improves the reliability of theoretical calculations. Moreover, the two $B$-factories experiments, Belle at KEKB and BaBar at PEP-II, have collected large data sample, sufficient to probe extensions of the Higgs sector and to provide meaningful constraints on parameter spaces in specific models.

Natural places to search for physics beyond the SM are processes which are suppressed in the SM. The flavor changing neutral current (FCNC) $B$ decays, being forbidden at the tree level and induced via loop diagrams in the SM, are widely recognized as a sensitive probe of new physics; in particular, radiative $b \rightarrow s \gamma$ decays play an important role in constraining the charged Higgs. Very promising observables can be accessed in leptonic and semileptonic $B$ decays to final states with a $\tau$ lepton, where the large $\tau$ mass enhances sensitivity to charged Higgs contribution at the tree level.

The main focus of this report are recent experimental results on $B^{+} \rightarrow \tau^{+} v_{\tau}{ }^{1}, B \rightarrow \bar{D}^{(*)} \tau^{+} v_{\tau}$ and inclusive $B \rightarrow X_{s} \gamma$ decays that currently provide the most stringent bounds on the charged Higgs sector.

Very high precision measurements of $\tau$ decays, which are being pursued at $B$-factories, can also give interesting indirect limits on $H^{ \pm}$, but they are beyond the scope of this paper [2].

\section{Experimental aspects}

The two $B$-factory experiments, Belle at KEKB and BaBar at PEP-II, have completed data taking with the total data samples containing 770 million $B \bar{B}$-pairs and 470 million $B \bar{B}$-pairs respectively, collected at the $\Upsilon(4 S)$ resonance. Most of the analyses presented in this report have not exploited the full data sets yet, still leaving some room for further improvement in accuracy.

Although technical details of studying inclusive $B \rightarrow X_{s} \gamma$ and multiple neutrino $B \rightarrow\left(D^{(*)}\right) \tau \nu_{\tau}$ decays are quite different, they share the common experimental challenge, which is the lack of clear-cut kinematic constraints. At $B$-factories experimental strategies exploit advantages of the exclusive production of $B \bar{B}$ pairs in the $\Upsilon(4 S)$ decays. The most straightforward approach is to reconstruct one of the two $B$-mesons $\left(B_{\text {tag }}\right)$ in a set of exclusive channels and to infer the decay of the other one $\left(B_{\mathrm{sig}}\right)$ from the remaining activity in the detector. The $B_{\text {tag }}$ candidates can be

\footnotetext{
${ }^{1}$ Charge conjugate modes are implied throughout the paper.
} 
selected using both hadronic and semileptonic $B$ decays. For hadronic tags, the $B_{\text {tag }}$ candidates are selected using the beam-energy-constrained mass and the energy difference variables: $M_{\text {tag }}=$ $\sqrt{E_{\text {beam }}^{2}-\mathbf{p}_{\text {tag }}^{2}}$ and $\Delta E_{\text {tag }}=E_{\text {tag }}-E_{\text {beam }}$, where $E_{\text {beam }}$ is the beam energy and $\mathbf{p}_{\text {tag }}$ and $E_{\text {tag }}$ denote the reconstructed momentum vector and energy of the $B_{\text {tag }}$ candidate in the $\Upsilon(4 S)$ rest frame. The semileptonic tag reconstruction exploits the cosine of the angle between $B$ and the $D^{(*)} \ell$ system which can be calculated as $\cos \Theta_{B-D^{(*)} \ell}=\left(2 E_{\text {beam }} E_{D^{(*)} \ell}-m_{B}^{2}-m_{D^{(*)} \ell}^{2}\right) /\left(2 p_{\text {beam }} p_{D^{(*)} \ell}\right)$ that takes values predominantly outside the range $[-1,1]$ for combinatorial $D^{(*)} \ell$ candidates.

Reconstruction of the $B_{\text {tag }}$ strongly suppresses combinatorial and continuum backgrounds and provides kinematic constraints on $B_{\text {sig. }}$. The main draw-back is the low $B_{\text {tag }}$ reconstruction efficiency, which is at the level of $10^{-3}$ for exclusive hadronic tags, and in many cases less restrictive constraints on the tagging side are imposed. Details of analyses are optimized individually for each process under study, compromising between the efficiency and purity of the signal. In particular, if a signal mode provides a clean signature, like a $D^{(*)}$ meson in semitauonic decays, the tagging efficiency can be increased several times by an "inclusive" reconstruction of $B_{\text {tag }}$ from all the particles that remain after selecting candidates for $B_{\text {sig }}$ daughters. As in exclusive tags, if there are no unpaired leptons on the tagging side, the $M_{\text {tag }}$ and $\Delta E_{\text {tag }}$ variables are used to check the consistency of a $B_{\text {tag }}$ candidate with a $B$-meson decay.

\section{3. $B^{+} \rightarrow \tau^{+} \nu_{\tau}$}

Purely leptonic decays $B^{+} \rightarrow \ell^{+} v_{\ell}$ proceed in the SM via $W$-mediated annihilation. The SM branching fraction is given by

$$
\mathscr{B}_{S M}\left(B^{+} \rightarrow \ell^{+} v_{\ell}\right)=\frac{G_{f}^{2} m_{B}}{8 \pi} m_{\ell}^{2}\left(1-\frac{m_{\ell}^{2}}{m_{B}^{2}}\right)^{2} f_{B}^{2}\left|V_{u b}\right|^{2} \tau_{B}
$$

where $G_{f}$ is the Fermi constant, $m_{\ell}(\ell=e, \mu, \tau)$ denotes the charged lepton mass, $V_{u b}$ is the CKM matrix element and $\tau_{B}$ and $m_{B}$ are the $B^{+}$lifetime and mass respectively. The $B$ meson decay constant, $f_{B}$, is a non-perturbative quantity to be estimated by theoretical methods like lattice QCD calculations. Due to the helicity suppression, represented by the factor $m_{\ell}^{2}$, the decay to $\tau^{+} v_{\tau}$ is most accessible experimentally with the expected branching fraction of the order of $10^{-4}$.

This decay rate can be significantly modified by a charged Higgs boson exchange. In the TypeII $2 \mathrm{HDM}$ the charged Higgs effect on the $\mathscr{B}\left(B \rightarrow \ell v_{\ell}\right)$ can be expressed as a multiplicative factor $r_{H}$, which is a function of $\tan \beta$ and charged Higgs boson mass, $m_{H^{ \pm}}[3]$ :

$$
r_{H} \equiv \frac{\mathscr{B}\left(B \rightarrow \ell v_{\ell}\right)}{\mathscr{B}\left(B \rightarrow \ell v_{\ell}\right)_{S M}}=\left(1-\tan ^{2} \beta \frac{m_{B}^{2}}{m_{H^{ \pm}}^{2}}\right)^{2}
$$

The $B^{+} \rightarrow \tau^{+} v_{\tau}$ decay rate represents a sensitive observable to probe $H^{ \pm}$except for very small values of $\tan \beta / m_{H^{ \pm}}$and around $\tan \beta / m_{H^{ \pm}}=\sqrt{2} / m_{B}$, where the $H^{ \pm}$contribution doubles that of the SM (with the opposite sign), and the factor $r_{H}$ takes values close to 1 (see Fig. 1).

Belle and BaBar performed searches of $B^{+} \rightarrow \tau^{+} v_{\tau}$ decays employing exclusive $B_{\text {tag }}$ reconstruction, both in hadronic and semileptonic modes. The signal yields are extracted from a fit to $E_{E C L}$, the energy of clusters in the electromagnetic calorimeter that are left after selecting the 
$B_{\text {tag }}$ and the $\tau$ daughter candidates. For signal events, $E_{E C L}$ peaks around zero, while background events tend to have higher values due to the contribution of additional clusters. The first evidence of the $B^{+} \rightarrow \tau^{+} v_{\tau}$ decay with a significance of 3.5 standard deviations $(\sigma)$ has been reported by Belle using hadronic tags [4]. The summary of the branching fraction measurements is presented in Table 1.

Table 1: Summary of $B^{+} \rightarrow \tau^{+} v_{\tau}$ branching fractions. The first branching fraction error is statistical and the second one is a systematic uncertainty. The significances of the signal excess, $\Sigma$, include systematic uncertainties.

\begin{tabular}{lclc}
\hline \multicolumn{1}{c}{ analysis } & data sample $(B \bar{B})$ & \multicolumn{1}{c}{$\mathscr{B} \times 10^{4}$} & $\Sigma(\sigma)$ \\
\hline \hline Belle, hadronic tags [4] & $449 \times 10^{6}$ & $1.79_{-0.49-0.51}^{+0.56+0.46}$ & 3.5 \\
Belle, semileptonic tags [5] & $657 \times 10^{6}$ & $1.54_{-0.37-0.39}^{+0.37}$ & 3.8 \\
BaBar, hadronic tags [6] & $468 \times 10^{6}$ & $1.80_{-0.54}^{+0.57} \pm 0.26$ & 3.3 \\
BaBar, semileptonic tags [7] & $459 \times 10^{6}$ & $1.8 \pm 0.8 \pm 0.2$ & 2.3 \\
\hline
\end{tabular}

The experimental results are systematically larger, but consistent within uncertainties, with the SM expectations. An illustration of the constraints in the $\left(m_{H^{ \pm}}, \tan \beta\right)$ parameter space are shown in Fig. 2 for $r_{H}=1.37 \pm 0.39$. The value of $r_{H}$ is evaluated with the current average experimental value of $\mathscr{B}\left(B^{+} \rightarrow \tau^{+} v_{\tau}\right)=(1.64 \pm 0.34) \times 10^{-4}$ [8] and the SM prediction $\mathscr{B}\left(B^{+} \rightarrow \tau^{+} v_{\tau}\right)_{S M}=$ $(1.20 \pm 0.25) \times 10^{-4}$, taking the $\left|V_{u b}\right|=(4.32 \pm 0.16 \pm 0.29) \times 10^{-3}$ from inclusive semileptonic $B$ decays [8] and $f_{B}=190 \pm 13 \mathrm{MeV}$ from lattice QCD calculations [9]. The bounds are derived within the Type-II 2HDM; however, they are only mildly modified by adding loop corrections in the MSSM (see e.g. [10]). On the other hand, the exclusion region can be appreciably shifted due to ambiguities in the SM prediction of the decay rate, coming from the choice of $f_{B}$ and systematic differences between $\left|V_{u b}\right|$ extracted from inclusive and exclusive methods. Moreover, an alternative approach to predict the SM value of the $\mathscr{B}\left(B^{+} \rightarrow \tau^{+} v_{\tau}\right)$ from the CKM fit using other measurements of the CKM parameters gives $\mathscr{B}\left(B^{+} \rightarrow \tau^{+} v_{\tau}\right)_{C K M}=\left(0.763_{-0.061}^{+0.114}\right) \times 10^{-4}$ [11], which is by $2.4 \sigma$ lower than the world average of direct measurements. This tension, though very interesting and deserving further study, represents an important issue in constraining charged Higgs sector from $B^{+} \rightarrow \tau^{+} v_{\tau}$.

4. $B \rightarrow D^{(*)} \tau v_{\tau}$

Semileptonic decays $B \rightarrow \bar{D}^{(*)} \tau^{+} v_{\tau}$ offer an alternative route to study charged Higgs effects at the tree level [12]. Having different sources of theoretical uncertainties, these decays are complementary to the $B^{+} \rightarrow \tau^{+} v_{\tau}$ mode. A broad spectrum of observables sensitive to new physics, like the $\tau$ polarization, will make the semitauonic $B$ decays competitive to the purely leptonic $B^{+} \rightarrow \tau^{+} v_{\tau}$ channel at future super B factories [13]. The effects of new physics are expected to be larger in $B \rightarrow \bar{D} \tau^{+} v_{\tau}$ than in $B \rightarrow \bar{D}^{*} \tau^{+} v_{\tau}$ [14]. It should be mentioned, however, that the decays to the vector meson are cleaner experimentally and offer an interesting possibility of studying correlations between the $D^{*}$ polarization and other observables [15].

The theoretical predictions for $B \rightarrow \bar{D}^{(*)} \tau^{+} v_{\tau}$ depend on the relatively well known element of the CKM matrix, $\left|V_{c b}\right|$, and on the form-factors, which are constrained by the Heavy Quark 


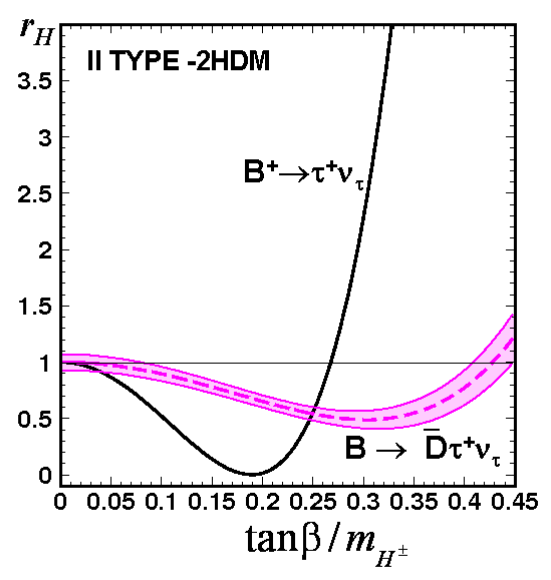

Figure 1: Charged Higgs effects in $B^{+} \rightarrow \tau^{+} v_{\tau}$ (black) [3] and $B \rightarrow \bar{D} \tau^{+} v_{\tau}$ (magenta, dashed) [17] decays. The shaded band around the $B \rightarrow \bar{D} \tau^{+} v_{\tau}$ curve indicates the combined uncertainties from theory and experimental error on the branching fraction of the reference $B \rightarrow \bar{D} \ell^{+} v_{\ell}$ mode.
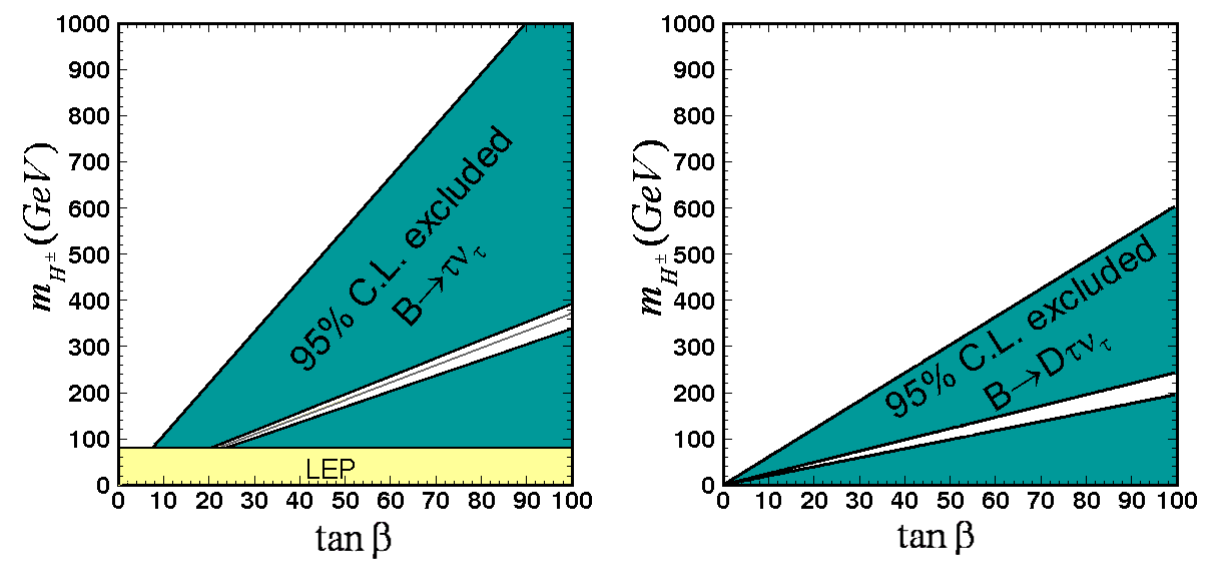

Figure 2: Constraints in the ( $\left.\tan \beta, m_{H^{ \pm}}\right)$plane for the Type-II $2 \mathrm{HDM}$ from $B^{+} \rightarrow \tau^{+} v_{\tau}$ (left) and $B^{\rightarrow} \bar{D} \tau^{+} v_{\tau}$ (right) decay rates. The shaded (green) areas are excluded at 95\% C.L.; the narrow allowed band corresponds to the $\tan \beta / m_{H^{ \pm}}$values, where the $H^{ \pm}$contribution is about twice as large as the SM one, and opposite in sign. For comparison, direct limits from LEP2 (yellow band) are superimposed on the left panel. 
Effective Theory [16] and by $B \rightarrow D^{(*)} \ell v_{\ell}(\ell=e, \mu)$ measurements. The dependence on hadronic parameters is further reduced in the ratio

$$
R_{D^{(*)} \ell v} \equiv \frac{\mathscr{B}\left(B \rightarrow D^{(*)} \tau v_{\tau}\right)}{\mathscr{B}\left(B \rightarrow D^{(*)} \ell v_{\ell}\right)}
$$

An example of expected charged Higgs effects in $B \rightarrow D \tau \nu_{\tau}$ mode [17] is presented in Fig. 1 together with the $B \rightarrow \tau \nu_{\tau}$ prediction, showing the complementarity of the two modes. Although the overall sensitivity of $B \rightarrow D \tau \nu_{\tau}$ is smaller than that of $B \rightarrow \tau \nu_{\tau}$, the semileptonic mode is superior in the region around $\tan \beta / m_{H^{ \pm}}=\sqrt{2} / m_{B}$.

Both Belle and $\mathrm{BaBar}$ reported results of searches of exclusive semitauonic $B$ decays. The decays are tagged with hadronic $B_{\text {tag }}$ decays reconstructed inclusively ([18],[19]) and exclusively ([20],[21]).

The signal candidates are selected by combining a $\bar{D}^{(*)}$ meson with a charged track expected from the $\tau^{+}$decay. The $\tau$ lepton candidates are reconstructed in leptonic modes $\tau^{+} \rightarrow e^{+} v_{e} \bar{v}_{\tau}$ ([18],[19],[20],[21]), $\tau^{+} \rightarrow \mu^{+} v_{\mu} \bar{v}_{\tau}$ ([19],[20],[21]) and in semileptonic decay $\tau^{+} \rightarrow \pi^{+} \bar{v}_{\tau}$ ([18], [19]). The latter is of special interest due to its sensitivity to the $\tau$ polarization.

The most discriminating variable, used to distinguish the signal from background, is the missing mass $M M$, corresponding to the effective mass of the neutrino system in the final state. In the analyses employing the exclusive $B_{\text {tag }}$ reconstruction, signal yields are extracted from fits to the two-dimensional distributions of $\left(M M^{2}, p_{\ell}\right)$ ( $p_{\ell}$ is the lepton momentum from $\tau$ decay) [20] and $\left(M M^{2}, E_{E C L}\right)$ [21]. In the inclusive $B_{\text {tag }}$ reconstruction the $M_{\text {tag }}$ variable is used to extract the signal. The $M_{\text {tag }}$ distribution is flat for most background components at large values of $M M^{2}$, while for the signal modes it shows a prominent peak at the $B$ mass. The fits are performed taking into account large cross-feeds between the $D^{*}$ and $D$ modes. In the analyses with exclusive tags, the results are normalized to the corresponding $B \rightarrow \bar{D}^{(*)} \ell^{+} v_{\ell}$ branching fractions to reduce a part of systematic uncertainties. The results of the measurements are summarized in Table 2.

The measured branching fractions can be compared with the SM expectations that are around $1.4 \%$ for $B \rightarrow \bar{D}^{*} \tau^{+} v_{\tau}$ and $0.7 \%$ for $B \rightarrow \bar{D} \tau^{+} \nu_{\tau}$ (see e.g.[23]).

An example of constraints on the $\tan \beta$ and $m_{H^{ \pm}}$parameters, obtained from the naïve average of $R_{D \ell v}=0.40 \pm 0.08$ [24] is shown in Fig. 2. Similarly to $B \rightarrow \tau v_{\tau}$, the $B \rightarrow D \tau v_{\tau}$ excludes two disjoint regions in the $\left(\tan \beta, m_{H^{ \pm}}\right)$plane, but the gaps are filled by combining the two modes. Further improvements in sensitivity can be achieved with larger data samples that will enable meaningful measurements of differential characteristics in semitauonic decays.

\section{5. $B \rightarrow X_{s} \gamma$}

The flavor changing neutral currents (FCNC), proceeding in the SM only at the loop level, offer a high sensitivity to new physics. In particular, inclusive $B \rightarrow X_{s} \gamma$ radiative decays with the partonic transition $b \rightarrow s \gamma$ have been very useful in constraining many new physics scenarios, including the charged Higgs sector. In addition to the penguin loops involving $W$ boson, new physics can also contribute at the one-loop level. In particular, the contribution of the charged Higgs loop adds constructively to the SM penguin, and is almost independent of $\tan \beta$, except for very small values, providing a very effective tool to constrain the $2 \mathrm{HDM}$ scenarios. On the other hand, additional new 
Table 2: Summary of the $B \rightarrow \bar{D}^{(*)} \tau^{+} v_{\tau}$ measurements. In the analyses [20] and [21] the branching fractions are evaluated from the $R_{D^{(*)} \ell v}$ measurements and the current average branching fractions of the normalization channels $B \rightarrow \bar{D}^{(*)} \ell^{+} v_{\ell}$ [22]. The quoted uncertainties are statistical (first), systematic (second) and due to the branching fraction error of the normalization mode (third). The significances of the excess, $\Sigma$, include systematic uncertainties.

\begin{tabular}{|c|c|c|c|c|}
\hline analysis & data sample $(B \bar{B})$ & $\mathscr{B} \times 10^{2}$ & $\Sigma(\sigma)$ & $R_{D^{(*)} \ell v}(\%)$ \\
\hline \multicolumn{5}{|c|}{ 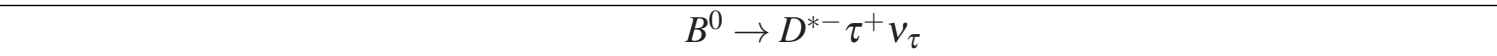 } \\
\hline Belle, incl. tags [18] & $535 \times 10^{6}$ & $2.02_{-0.37}^{+0.40} \pm 0.37$ & 5.2 & \\
\hline Belle, excl. tags [21] & $657 \times 10^{6}$ & $2.56_{-0.66-0.22}^{+0.75+0.31} \pm 0.10$ & 4.7 & $48_{-12-4}^{+14+6}$ \\
\hline BaBar, excl. tags [20] & $232 \times 10^{6}$ & $1.11 \pm 0.51 \pm 0.04 \pm 0.04$ & 2.7 & $20.7 \pm 9.5 \pm 0.8$ \\
\hline \multicolumn{5}{|c|}{$B^{+} \rightarrow \bar{D}^{* 0} \tau^{+} v_{\tau}$} \\
\hline Belle, incl. tags [19] & $657 \times 10^{6}$ & $2.12_{-0.27}^{+0.28} \pm 0.29$ & 8.1 & \\
\hline Belle, excl. tags [21] & $657 \times 10^{6}$ & $3.04_{-0.66-0.47}^{+0.69+0.40} \pm 0.22$ & 3.9 & $47_{-10-7}^{+11+6}$ \\
\hline BaBar, excl. tags [20] & $232 \times 10^{6}$ & $2.25 \pm 0.48 \pm 0.22 \pm 0.17$ & 5.3 & $34.6 \pm 7.3 \pm 3.4$ \\
\hline \multicolumn{5}{|c|}{$B^{0} \rightarrow D^{-} \tau^{+} v_{\tau}$} \\
\hline Belle, excl. tags [21] & $657 \times 10^{6}$ & $1.01_{-0.41-0.11}^{+0.46+0.13} \pm 0.10$ & 2.6 & $48_{-19-5}^{+22+6}$ \\
\hline BaBar, excl. tags [20] & $232 \times 10^{6}$ & $1.04 \pm 0.35 \pm 0.15 \pm 0.10$ & 3.3 & $48.9 \pm 16.5 \pm 6.9$ \\
\hline \multicolumn{5}{|c|}{$B^{+} \rightarrow \bar{D}^{0} \tau^{+} v_{\tau}$} \\
\hline Belle, incl. tags [19] & $657 \times 10^{6}$ & $0.77 \pm 0.22 \pm 0.12$ & 3.5 & \\
\hline Belle, excl. tags [21] & $657 \times 10^{6}$ & $1.51_{-0.39-0.19}^{+0.41+0.24} \pm 0.15$ & 3.8 & $70_{-18-9}^{+19+11}$ \\
\hline BaBar, excl. tags [20] & $232 \times 10^{6}$ & $0.67 \pm 0.37 \pm 0.11 \pm 0.07$ & 1.8 & $31.4 \pm 17.0 \pm 4.9$ \\
\hline
\end{tabular}

physics contributions, such as squark-chargino loops in SUSY models, make an interpretation of the measurements more ambiguous.

The calculation of $\mathscr{B}\left(B \rightarrow X_{s} \gamma\right)$ has been completed up to next-to-next-to leading order (NNLO) [25], reducing substantially the theoretical uncertainty on the inclusive branching fraction prediction. The predicted value for the photon energy cut $E_{\gamma}>1.6 \mathrm{GeV}$ is $\mathscr{B}\left(B \rightarrow X_{s} \gamma\right)_{E_{\gamma>1.6 \mathrm{GeV}}}=$ $(3.15 \pm 0.23) \times 10^{-4}$.

Experimental efforts aim at making the most inclusive measurements possible in order to minimize the model dependence in comparing the results with the theoretical calculations. The easiest approach, based on summing a large number of fully reconstructed exclusive radiative decay channels, suffers from large systematic uncertainties associated with the corrections for unseen modes. An alternative approach is to identify the $B \rightarrow X_{s} \gamma$ event by tagging the high energy photon, with minimal requirements on the $X_{s}$ system. Much effort is devoted to lowering the photon energy threshold. The large coverage of the photon spectrum is essential to reduce the theory uncertainties, but results in an overwhelming background from low energy photons. The dominant background source coming from the continuum quark pair production is subtracted using data taken below the $\Upsilon(4 S)$ resonance. Backgrounds from $\pi^{0} \rightarrow \gamma \gamma$ and $\eta \rightarrow \gamma \gamma$ from $B$ decays are evaluated from measurements of $\pi^{0}$ and $\eta$ spectra produced in $B$ decays.

There are several possibilities to reduce the continuum background. They are based on the characteristics of $B_{\text {tag }}$ that provide at the same time information on the flavor of $B_{\text {sig }}$, but at the expense of a substantial signal reduction. For the data samples currently available, the best results 
are obtained by tagging with high momentum leptons.

Experiments operating at the $\Upsilon(4 S) e^{+} e^{-}$colliders provided a set of inclusive $B \rightarrow X_{s} \gamma$ measurements employing various techniques. The latest measurement by Belle combines untagged and lepton tagged samples extracted from $657 \times 10^{6} B \bar{B}$-pairs [26]. Large statistics and improved experimental techniques allowed to lower the photon energy threshold down to $1.7 \mathrm{GeV}$, covering for the first time more than $97 \%$ of the $B \rightarrow X_{s} \gamma$ phase space, and provided the most precise measurement of the partial branching fraction $\mathscr{B}\left(B \rightarrow X_{s} \gamma\right)_{E_{\gamma}>1.7 \mathrm{GeV}}=(3.45 \pm 0.15 \pm 0.40) \times 10^{-4}$, where the errors are statistical and systematic.

The average of experimental measurements, extrapolated to the partial branching fraction for $E_{\gamma}>1.6 \mathrm{GeV}$, yields $\mathscr{B}\left(B \rightarrow X_{s} \gamma\right)=(3.55 \pm 0.24 \pm 0.09) \times 10^{-4}$ [8] which is marginally consistent with the SM prediction. This result provides a 95\% C.L. lower bound for the charged Higgs mass $m_{H^{ \pm}}>295 \mathrm{GeV}$ in the Type-II 2HDM [25].

\section{Summary and outlook}

$B$ physics observables represent a sensitive probe of the charged Higgs sector and current constraints on $H^{ \pm}$are dominated by indirect measurements at $B$-factories. The most robust limits are placed on the parameters $\tan \beta$ and $M_{H^{ \pm}}$of the two-Higgs-doublet model of Type-II. The energy scale probed in these measurements surpasses current direct searches at the Tevatron, and the obtained bounds overlap with the region of the highest sensitivity to the charged Higgs discovery at the LHC.

Further improvements in measurements of the $B$ physics observables, especially the $B^{+} \rightarrow$ $\tau^{+} v_{\tau}$ and $B \rightarrow \bar{D} \tau^{+} v_{\tau}$ branching fractions, can be achieved rather quickly using the full data samples collected at the $B$-factories and by applying more efficient tagging techniques. The potential of flavor physics for studying the charged Higgs sector will be greatly enhanced at the future super B factories.

\section{References}

[1] J.F. Gunion, H.E. Haber, G. Kane and S. Dawson, “The Higgs Hunter's Guide”, Addison-Wesley, New York U.S.A. (1990).

[2] S. Towers, hep-ex/0004022.

[3] W. Hou, Phys. Rev. D 48,2342(1993).

[4] K. Ikado et al. (Belle collaboration), Phys. Rev. Lett. 97, 251802 (2006).

[5] K. Hara et al. (Belle collaboration), Phys. Rev. D 82, 071101(R) (2010).

[6] B. Aubert et al. (BaBar collaboration), arXiv:1008.0104 [hep-ex].

[7] B. Aubert et al. (BaBar collaboration), Phys. Rev. D 81, 051101 (2010).

[8] D. Asner et al. (Heavy Flavor Averaging Group), arXiv:101.1589v1 [hep-ex] and http://www.slac.stanford.edu/xorg/hfag/.

[9] E. Gámiz et al. (HPQCD collaboration), Phys. Rev. D 80, 014503 (2009).

[10] G. Isidori, Nuclear Physics B - Proceedings Supplements, 163, 192 (2007). 
[11] S. T'Jampens (CKMfitter Group), talk presented at 35th International Conference on High Energy Physics (ICHEP10), July 21-28, 2010, Paris, France.

[12] A. S. Cornell et al., Phys. Rev. D 81, 094018 (2010) and references quoted therein.

[13] T. Abe et al., Belle II Technical Design Report, KEK Report 2010-1, July 2010, M. Bona et al., SuperB: A High-Luminosity Asymmetric $e^{+} e^{-}$Super Flavor Factory. Conceptual Design Report., arXiv:0709.0451 [hep-ex].

[14] M. Tanaka, Z. Phys. C 47, 321 (1995).

[15] R. Garisto, Phys. Rev. D 51, 1107 (1995).

[16] N. Isgur and M. B. Wise, Phys. Lett. B 5232, 113 (1989).

[17] J. F. Kamenik and F. Mescia, Phys. Rev. D 78, 014003 (2008).

[18] A. Matyja et al. (Belle collaboration), Phys. Rev. Lett. 99, 191807 (2001).

[19] A. Bozek et al. (Belle collaboration), Phys. Rev. D 82, 072005 (2010).

[20] B. Aubert et al. (BaBar collaboration), Phys. Rev. Lett. 100, 021801 (2008), Phys. Rev. D 79, 092002 (2009).

[21] I. Adachi et al. (Belle collaboration), arXiv:0910.430 [hep-ex].

[22] W. M. Yao et al. (Particle Data Group), J. Phys. G 33, 1 (2003).

[23] C.-H. Chen and C.-Q. Geng, JHEP 0610, 053 (2006).

[24] M. Tanaka and R. Watanabe, Phys. Rev. D 82, 034027 (2010).

[25] M. Misiak et al., Phys. Rev. Lett. 98, 022002 (2007).

[26] A. Limosani et al. (Belle collaboration), Phys. Rev. Lett. 103, 241801 (2009). 